\title{
NOTE ON A PROBLEM IN NUMBER THEORY
}

\author{
HAROLD N. SHAPIRO
}

The problem which we shall consider originated from a conjecture of S. Ulam. For $x, p$, integers, $p$ a prime, let $x \equiv a(\bmod p)$ where $-p / 2<a<p / 2$; and define $\|x\|_{p}=|a|$. Then if $T(x)$ is a mapping of the nonzero residues modulo $p$ into themselves, we consider the following "approximate multiplicative relation" modulo $p$,

$$
\|T(x y)-T(x) T(y)\|_{p}<k
$$

where $k$ is a fixed integer. The problem is to ascertain simple conditions under which the only solutions to (1) are given by

$$
T(x) \equiv x^{a}(\bmod p) .
$$

Clearly, $p$ must be larger than $k$ in order that this be feasible. Also, if we give to $T(x)$ any arbitrary set of integral values between 0 and $k^{1 / 2}$ we may obtain mappings satisfying (1) but not (2). This then indicates in a sense that the value domain of $T(x)$ must not be too small in order that (2) follow from (1).

The results obtained in this note are derived essentially from the following very simple lemma.

LEMMA. If for $T(x)$ a mapping of a semigroup $G$ into a ring $R$ we define

$$
\epsilon(x, y)=T(x y)-T(x) T(y)
$$

then for any $x, y, z$ of $G$,

$$
\epsilon(x, y) T(z)+\epsilon(x y, z)=T(x) \epsilon(y, z)+\epsilon(x, y z) .
$$

Proof. For any $x, y, z$ of $G$ we obtain from the associativity of multiplication:

$$
\begin{aligned}
T(x y z) & =T(x y) T(z)+\epsilon(x y, z) \\
& =T(x) T(y) T(z)+\epsilon(x, y) T(z)+\epsilon(x y, z)
\end{aligned}
$$

and

$$
\begin{aligned}
T(x y z) & =T(x) T(y z)+\epsilon(x, y z) \\
& =T(x) T(y) T(z)+T(x) \epsilon(y, z)+\epsilon(x, y z) .
\end{aligned}
$$

Comparing (5) and (6) yields (4).

Received by the editors October 30,1947, and, in revised form, November 10, 1947. 
We note that in case both $G$ and $R$ are commutative, as they will be in our applications of the lemma, we have $\epsilon(x, y)=\epsilon(y, x)$ and we may write (4) as

$$
\epsilon(x, y) T(z)+\epsilon(z, x y)=\epsilon(y, z) T(x)+\epsilon(x, y z) .
$$

To begin with we shall consider the case where $T(x)$ is a mapping of the residues modulo $n$, which are prime to $n$, into themselves, where $n$ is not necessarily prime. In this case we have the following theorem.

THEOREM 1. If (1) $\|T(x y)-T(x) T(y)\|_{n}<k$, (2) $k<\min _{p \mid n} p$, and (3) $T(x)$ takes on more than $8 k^{2}$ distinct values, then

$$
T(x y) \equiv T(x) T(y)(\bmod n) .
$$

Proof. Suppose that, for some two integers $x_{1}, x_{2}$, (8) does not hold, so that

$$
T\left(x_{1} x_{2}\right) \equiv T\left(x_{1}\right) T\left(x_{2}\right)+\epsilon\left(x_{1}, x_{2}\right)(\bmod n)
$$

where

$$
\left\|\epsilon\left(x_{1}, x_{2}\right)\right\|_{n}<k
$$

and $\epsilon\left(x_{1}, x_{2}\right) \not \equiv 0(\bmod n)$.

We now apply the lemma to the case where $G$ is the group of residues $\bmod n$ which are prime to $n$, and $R$ all residues. We get for any integer $y$,

$$
\epsilon\left(x_{1}, x_{2}\right) T(y)+\epsilon\left(y, x_{1} x_{2}\right) \equiv \epsilon\left(x_{2}, y\right) T\left(x_{1}\right)+\epsilon\left(x_{1}, x_{2} y\right)
$$

where from hypothesis (1) we have $\left\|\epsilon\left(y, x_{1} x_{2}\right)\right\|_{n}<k, \| \epsilon\left(x_{2}, y \|_{n}<k\right.$, and $\left\|\epsilon\left(x_{1}, x_{2} y\right)\right\|_{n}<k$. From the hypothesis $k<\min _{p \mid n} p$ so that by (9) we see that $\left(\epsilon\left(x_{1}, x_{2}\right), n\right)=1$. Thus having fixed $x_{1}, x_{2}, \epsilon\left(x_{1}, x_{2}\right)$ is fixed, and (10) determines the value of $T(y)$ uniquely, modulo $n$. Clearly then $T(y)$ could take on at most $8 k^{2}$ distinct values, whence the theorem.

For the special case where $n=p^{\alpha}$, the power of an odd prime we get the following theorem.

THEOREM 2. If (1) $\|T(x y)-T(x) T(y)\|_{p^{\alpha}}<k$, (2) $k<p$, and (3) $T(x)$ takes on more than $8 k^{2}$ distinct values,

$$
T(x) \equiv x^{a}\left(\bmod p^{\alpha}\right) .
$$

Proof. From Theorem 1 we see that $T(x y) \equiv T(x) T(y)\left(\bmod p^{\alpha}\right)$. Then if $\rho$ is a primitive root $\bmod p^{\alpha}$ we have 


$$
T(x) \equiv T\left(\rho^{\mu}\right) \equiv\{T(\rho)\}^{\mu} \equiv \rho^{a \mu} \equiv x^{a}\left(\bmod p^{\alpha}\right) .
$$

Corollary. If $\|T(x y)-T(x) T(y)\|_{p}<k$ and $T(x)$ takes on more than $8 k^{2}$ distinct values, then $T(x) \equiv x^{a}(\bmod p)$.

If we now restrict our attention to the case of a prime modulus we see that the above discussion covers those cases for which $N=$ the number of distinct values in the value domain is such that $N<k^{1 / 2}$ or $N>8 k^{2}$. The question then arises as to what can be said for $k^{1 / 2}<N$ $\leqq 8 k^{2}$. In this direction we have:

THEOREM 3. If $N>8 k$, and $\|T(x y)-T(x) T(y)\|_{p}<k$, then, for $p>8 k^{2}$, $N$ divides $p-1$.

Proof. (a) We first note that $T(1)=1$. For we have for any $x$

$$
T(x)(1-T(1))=\epsilon(1, x), \quad\|\epsilon(1, x)\|_{p}<k,
$$

so that if $T(1) \neq 1, T(x)$ could take on at most $2 k$ values.

(b) If $N>8 k^{2}$ we have already proved above that $T(x) \equiv x^{a}(\bmod p)$ and it follows in this case that $N \mid(p-1)$.

(c) It remains only to consider the case $8 k<M \leqq 8 k^{2}$.

Since $N \leqq 8 k^{2}$ there must be a value $c$ such that $T(x)=c$ for more than $(p-1) / N$ different $x$. Let $z_{1}, \cdots, z_{\Delta}, \Delta \geqq(p-1) / N$, be the numbers such that $T\left(z_{i}\right)=c, i=1, \cdots, \Delta$. Then for $p$ sufficiently large (for example, $\left.p>8 k^{2}\right) \Delta>1$, and we have from (7), for $i \neq j$,

$$
\begin{aligned}
& \epsilon(x, y) T\left(z_{i}\right)+\epsilon\left(z_{i}, x y\right) \equiv \epsilon\left(x, z_{i}\right) T(y)+\epsilon\left(y, z_{i} x\right), \\
& \epsilon(x, y) T\left(z_{j}\right)+\epsilon\left(z_{j}, x y\right) \equiv \epsilon\left(x, z_{j}\right) T(y)+\epsilon\left(y, z_{j} x\right) .
\end{aligned}
$$

Subtracting (11) and (12) gives for all $x, y, i, j$,

$$
\begin{aligned}
\epsilon\left(z_{i}, x y\right)-\epsilon\left(z_{j}, x y\right) \equiv & T(y)\left\{\epsilon\left(x, z_{i}\right)-\epsilon\left(x, z_{j}\right)\right\} \\
& +\epsilon\left(y, z_{i} x\right)-\epsilon\left(y, z_{j} x\right) .
\end{aligned}
$$

If for some $x, i, j, \epsilon\left(x, z_{i}\right) \not \equiv \epsilon\left(x, z_{j}\right),(13)$ implies that $T(y)$ has a value domain of not more than $8 k$ values, which contradicts the hypothesis. Hence for all $x ; i, j=1, \cdots, \Delta$,

$$
\epsilon\left(x, z_{i}\right)=\epsilon\left(x, z_{j}\right) .
$$

This gives $T\left(x z_{i}\right)-T(x) T\left(z_{i}\right)=T\left(x z_{j}\right)-T(x) T\left(z_{j}\right)$ or

$$
T\left(x z_{i}\right)=T\left(x z_{j}\right) .
$$

Replacing $x$ by $x z_{i}^{-1}$ in (15) we get for all $x, i, j=1, \cdots, \Delta$,

$$
T(x)=T\left(x z_{i}^{-1} z_{j}\right) \text {. }
$$


From (16) we see that $Z=\left\{z_{1}^{-1} z_{1}, z_{1}^{-1} z_{2}, \cdots, z_{1}^{-1} z_{\Delta}\right\}$ are $\Delta$ distinct numbers such that

$$
T\left(z_{1}^{-1} z_{i}\right)=1 .
$$

Then if $\alpha_{1}, \cdots, \alpha_{N}$ are the $N$ distinct values in the value domain and $x_{1}, \cdots, x_{N}$ are such that $T\left(x_{i}\right)=\alpha_{i}, i=1, \cdots, N$, we see from (16) that

$$
T\left(x_{i} Z\right)=T\left(x_{i}\right)=\alpha_{i}, \quad i=1, \cdots, N .
$$

Hence at least $\Delta$ numbers map into each $\alpha_{i}$, and we have $p-1 \geqq \Delta N$ $\geqq p-1$. Thus $p-1=N \Delta$ and $N \mid(p-1)$.

Corollary 1. If $N>8 k, p>8 k^{2}$, and $\|T(x y)-T(x) T(y)\|_{p}<k$, then the set $Z$ of those numbers which map into 1 is a subgroup of order $(p-1) / N$ of the group of residues prime to $p$, and all the elements of $a$ given coset map into the same number.

Proof. To prove that $Z$ is a group we need only show that it is closed. Both this and the remainder of the corollary is clear from (16) and Theorem 2.

Corollary 2. If $N>8 k, p>8 k^{2},\|T(x y)-T(x) T(y)\|_{p}<k$, and $p-1$ has no divisors which lie in the interval $\left(8 k, 8 k^{2}\right)$, then $T(x) \equiv x^{a}(\bmod p)$.

Corollary 3. If $\|T(x y)-T(x) T(y)\|_{p}<k, k>0, p>8 k^{2}$ and, for some primitive root $\rho, T(\rho)=1$ then $N \leqq 8 k$.

Proof. If $N>8 k$ then the subgroup $Z$ contains $\rho$ and hence $Z$ is the whole group. But this means $N=1>8 k$ which is impossible.

Princeton University 